\title{
Creative Activity Support by Discovering Effective Combinations
}

\author{
Yoko Nishihara, ${ }^{1}$ Wataru Sunayama, ${ }^{2}$ and Masahiko Yachida ${ }^{1}$ \\ ${ }^{1}$ Graduate School of Engineering Science, Osaka University, Toyonaka, 560-8531 Japan \\ ${ }^{2}$ Faculty of Information Sciences, Department of Information Machines and Interfaces, Hiroshima, 731-3194 Japan
}

\section{SUMMARY}

Creative activities based on combinations are now being carried on everywhere in the world. Whether or not the creative activity succeeds depends on the combinations used in it. Since there are tremendous numbers of candidate combinations, it is impossible to decide at a glance which should be used. Thus, there must be a method of efficiently discovering combinations which are effective in making creative activity a success. This paper proposes a method of discovering combinations that is effective in supporting creative activity. In the proposed method, the originality of each combination and the novelty perceived by users are estimated from the number of hits on Web pages containing the keywords in question, and the effectiveness of the combinations is judged. (c) 2007 Wiley Periodicals, Inc. Syst Comp Jpn, 38(12): 99-111, 2007; Published online in Wiley InterScience (www.interscience.wiley.com). DOI 10.1002/scj.20392

Key words: creative activity; combination; prevalence prediction.

\section{Introduction}

Creative activities based on combinations are occurring everywhere in the world. As can be seen when "TV phone" is approximately represented as a combination of "TV" and "phone," most objects in the world can be approximately represented as combinations of existing objects. This principle is likely to apply to many objects which will be created in the future.

Anyone can attempt creative activity by combination. However, its success or failure depends on various factors related to the combination under consideration.

Consequently, there must be a thorough investigation of the effectiveness of combinations in creative activity. Since there is a tremendous number of possible combinations in the world, it is difficult to tell at a glance which combinations will be useful. It is also impossible to investigate all combinations due to limitations of time and labor.

Furthermore, creative activity does not succeed unless a combination is useful for the purpose of the activity. Generally, when a combination is of a usual kind for creative activity, it is highly probable that the result will be accepted by many users. When the combination is of an unusual nature, the probability that it will be accepted by many users is low. On the other hand, since the approach is original, the probability that a combination already exists for that activity is low. Consequently, such a combination should be highly evaluated as a new approach to achieving the objective. In other words, it is very useful to discover a combination which is original and has a high possibility of being accepted by many users.

Based on such reasoning, this paper treats new concepts as combinations of existing objects. A system which presents new original combinations that are highly likely to

(C) 2007 Wiley Periodicals, Inc. 
become established is proposed in order to support creative activity by improving human efficiency in examining combinations. That is, the purpose of this study is not to positively support humans in conceiving ideas that can give rise to new concepts or creative thought, but to present useful combinations of existing concepts as material to help humans generate ideas.

Below, Section 2 describes related studies and characterizes this study. Section 3 describes a preliminary experiment on the evaluation of combinations. Section 4 describes the proposed combination evaluation system. Section 5 compares the system's output with combinations that have already begun to win acceptance. It also compares the proposed system with conventional methods. Section 6 concludes the paper.

\section{Related Work and Characterization of This Study}

There have been many efforts to support human creative activity, with various purposes of activity and means of support. There is a study of knowledge creation support in which the activity is supported by dialog generation [1]. In this system, a dialog is generated between the user and an avatar. An avatar is an agent with the function of representing the user's information in a computer realization. In the dialog generated by the avatar, the responses do not include any profound semantic processing, but the user can interpret the utterances of the agent as meaningful with a natural feeling. Knowledge is constructed through such a process.

There is a program called ELIZA that supports creative activity through dialog, in which a natural conversation is realized between a human and a computer [2]. ELIZA extracts the words contained in the input sentence and constructs response sentences based on response templates stored in the database. An effort at creative dialog is made by using the function of adding a response template and the function of learning the segmentation of the sentence implications.

There have been efforts to use combinations of objects in supporting creative activity. For example, Takasugi and Kunifuji [3] considered that new ideas are generated from combinations, while the user conceives of various kinds of information. An attempt is made to support the idea by arranging keywords and texts which are closely related to a keyword input by the user on a two-dimensional plane. In this study too it is conjectured that the creation of new ideas is based on existing concepts, and an attempt is made to support the activity of creating new ideas by association based on combinations.
This paper considers the following aspects of the activity of creating new combinations:

(1) Formulation of research activity and titles of papers in research activity

(2) Development of new products for sale in convenience stores

In item (1), combinations to be embodied in research activity which will establish itself in the future are predicted. In item (2), combinations to be embodied in products which will be successful in convenience stores are anticipated. In the human anticipation of success, an idea often starts with an object which will be successful, or an object which has long been successful.

There is a study in which the prediction of future success is supported by outputting important sentences including a keyword of interest [4]. In this approach, the novelty of the information concerning the keyword or the relation to the user's viewpoint is inferred from data on the Web, and keywords which are increasingly being searched for are extracted. The extracted keywords and important sentences including them in the text data on the Web are output.

It is difficult to predict success by simply presenting keywords. By presenting important sentences as examples of the use of the keywords, however, it becomes easier for the user to predict success from keywords. But it should be noted that the presented keyword is intended only to provide a starting point for predictions of success, from which the user must continue with the prediction process. This paper differs from the above approach by focusing on prediction of objects which have a higher likelihood of success.

There is also a study in which keywords and concepts which are expected to win acceptance are directly predicted [5]. The approach in this study is based on the idea that there should be some social factor behind success. The social factor is represented by the word, and the semantic distance to a word which is likely to win acceptance in the future is determined. It is inferred that an object is more likely to win acceptance if the distance is smaller. The above approach is similar to this study in that a newly created object is predicted. The distance is determined by applying the vector space method, familiar in natural language processing, to descriptive sentences attached to each word. In fact, the distance does not differ much, since the description sentences are short. Thus, what is actually predicted is a field related to keywords which will win acceptance in the future.

As regards the prediction of future emergence, there has been an effort to predict the generation of earthquakes by using the method of deriving assertions from documents [6]. In this study, the past history of earthquakes is treated as a document, and dislocations with stresses are found by 
applying a system called KeyGraph [7] to earthquake prediction. KeyGraph was originally proposed as a method in which the semantic structure of documents is represented as a graph and keywords containing the assertions of the document are extracted. The method presented in this paper differs in the following respects. For two keywords, the changes of recognition and the deviation from the joint probability of occurrence are determined, and the future popularity of the object to be newly constructed is predicted.

In this paper, combinations are considered in connection with creative activity. It has been observed in various problems that new objects are approximately represented by combinations. As can be seen in the case of the TV phone discussed above, most of the objects in daily life can be represented by a combination of multiple objects. In branding strategy [8], which is a management strategy of business organizations, new products are developed on the basis of combinations.

It is understood that the important feature of the brand strategy is to convey a different impression to the customer. In order to create a difference in image, companies try to develop new products by combining a product under its own brand with a product of another company. The new product in this case is created by line extension of the company's own branded product and category extension of the other company's product. By line extension is meant the development of a new product by adding a new function while retaining the essence of the product despite the extension. By category extension is meant the development of a new product by adding a function already present in the object used for extension to another object.

In the process, what is developed as a result of combination is essentially inheritance of the object on the line extension side, and inclusion of the function on the category extension side as additional value. Both line extension and category extension can be applied to any object, and different products will result according to the variety of extensions.

Examples of the brand strategy are as follows.

- Disney Sea: line extension of "Disneyland" + category extension of "Sea"

- Print Club: line extension of "game" + category extension of "photo"

- Portable phone with camera: line extension of "portable phone" + category extension of "camera"

In this study, a "combination" is defined as consisting of the line extension of keyword $L$ and the category extension of keyword $C$.

\section{Preliminary Experiment on Evaluation of Combinations}

This section describes a preliminary experiment on the evaluation of combinations. The method described in the previous section is one of the methods used by a business firm in a branding strategy in order to establish its own brand in a new product developed by line extension of one of its existing products and category extension of another product. Many successful products consist of approximate combinations of these two.

A product that is to be successful should have some factors promoting success. Consequently, the degree of attention to an object is defined as the number of users who have an interest in that object. The degree of attention can then be estimated by the number of hits on a keyword representing that object (or the number of data containing the keyword). Consequently, the degrees of attention that are reflected in the respective hit numbers of the keyword of the line extension side and the keyword of the category extension side are determined. The factors for success are then estimated from the behavior of the degree of attention.

As the first step in the experiment, 2627 noun keywords were extracted from the rental diary site "Sarusaru diary" [9]. The number of hits on each keyword and the number of coincident hits on two different keywords were examined in each month from March 2000 to May 2003. Then, for a product composed of a combination which became successful, the keyword of the line extension side and the keyword of the category extension side were considered. The number of hits on the two keywords and the number of coincident hits ${ }^{\dagger}$ were examined for 3 months before and after the first marketing of the product. The tendency exhibited by these data was investigated.

As examples, Figs. 1 and 2 show the tendencies of the number of hits on 8 products. The vertical axes in Figs. 1 and 2 were derived as follows. The average rate of increase of the number of coincident hits in the 3 months after the start of sales of each product was divided by the average rate of increase of all combinations plus the standard deviation. ${ }^{\ddagger}$ The value on the horizontal axis in Fig. 1 was obtained by dividing the average number of hits in the 3 months before and after the start of sales of each product by the

\footnotetext{
${ }^{\dagger}$ The number of data containing both the keyword of the line extension side and the keyword of the category extension side.

${ }^{*}$ The data were normalized so that the mean plus the standard deviation was 1 . It was verified experimentally that the rates of increase of the number of hits on the keyword of the line extension side, the number of hits on the keyword of the category extension side, and the number of coincident hits on the two keywords all followed a normal distribution. Normalization was applied so that the top data in the distribution above the mean + standard deviation (top 16\% in the normal distribution) could easily be identified as values above 1 .
} 


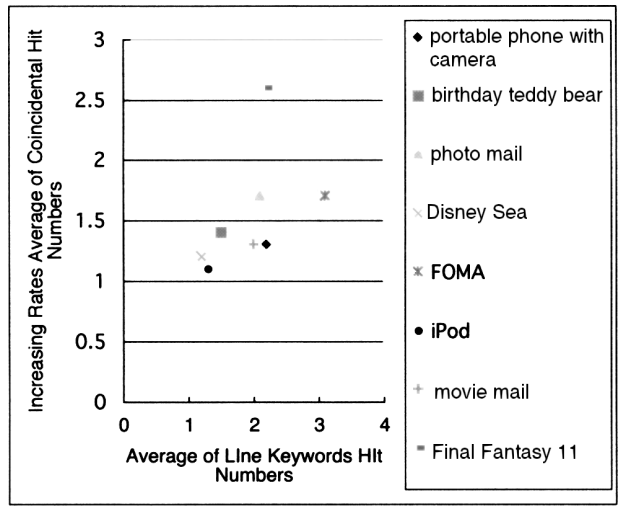

Fig. 1. Hit numbers of line keywords and rates of coincidental hit numbers.

average number of hits on all keywords plus the standard deviation. The value on the horizontal axis in Fig. 2 was obtained by dividing the average rate of increase of the number of hits in the 3 months before the start of the sales of each product by the average rate of increase of the number of hits on all keywords plus the standard deviation.

It is evident from Figs. 1 and 2 that the rates of increase of the number of hits on the keyword of the line extension side, the number of hits on the keyword of the category extension side, and the number of coincident hits on the two keywords were high for the successful products. The fact that the number of hits on a keyword is higher than the average plus the standard deviation implies that the recognition of the keyword is fairly high. The fact that the relative number of hits on the keywords is higher than the average plus the standard deviation implies that the keyword has novelty. It is thus evident that a product composed of a successful combination has the properties that the

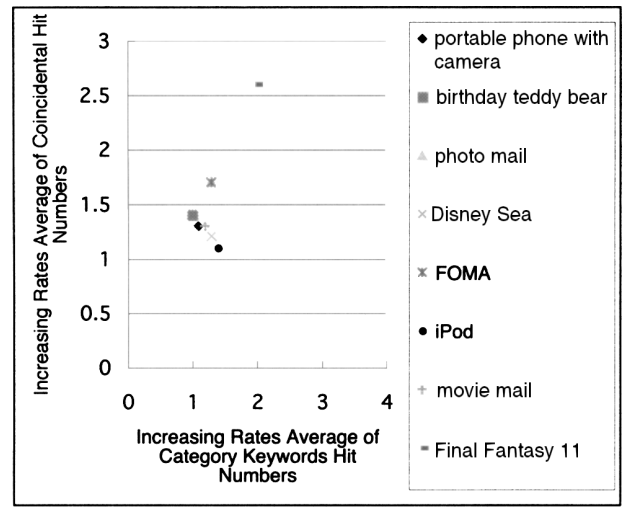

Fig. 2. Hit numbers of category keywords and rates of coincidental hit numbers. object represented by the keyword of the line extension side is somewhat recognized and the object represented by the keyword of the category extension side has novelty.

\section{Combination Evaluation System}

This section describes the evaluation system proposed in this paper. Figure 3 shows the configuration of the system. The preliminary experiment presented in Section 3 showed that a combination approximately representing a product that succeeds has the properties that the keyword of the line extension side is recognized to some extent and the keyword of the category extension side has novelty. Consequently, the system is designed so that the user inputs a recognized keyword from the line extension side, and the system outputs a keyword of the category extension side representing an object with novelty.

The system receives the keyword $L$ representing the object of the line extension side as the input. Then a combination is formed with the keyword $C$ representing an object in the database on the category extension side. For the combination thus constructed, the evaluation values representing respectively the originality of combination and the potential popularity (novelty) of the combination are calculated. Finally, the evaluation score for the combination is derived from the two different evaluation values. The keywords $C$ of the category extension side that form an effective combination are output in order of their evaluation scores. The two keyword databases and the system modules prepared in the proposed system are described below.

\subsection{Keyword database}

Two databases are contained in the proposed system. Each database contains the keywords to be used in combinations, the number of hits on each keyword at a certain time, and the number of coincident hits on two different keywords, for a period of several years. One database is used to predict the titles of papers, and the other database

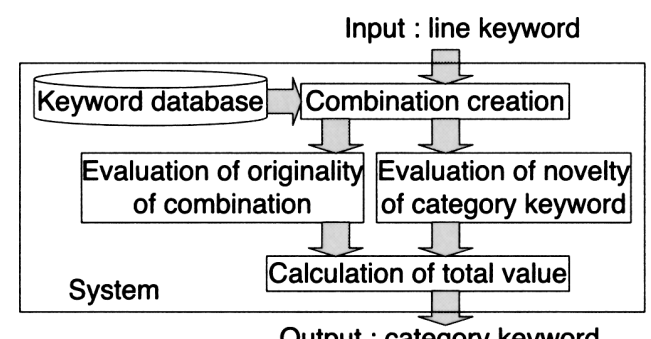

Fig. 3. Evaluation system of combinations. 
is used to predict new products in convenience stores. The procedure for preparing the set of keywords for each database and the details of each database are described below.

\subsubsection{Database for paper titles}

There are many fields of research in the world and many societies related to these fields. Keywords indicating the content of research differ depending on the research field. For example, many papers presented to the Society for Artificial Intelligence treat data mining, but few discuss elementary particles. Furthermore, the keywords which are used in research activity are technical terms, differing from words used in daily conversation. Consequently, rather than collecting all conceivable keywords, the keywords are limited to those which are somewhat specialized, and noise is thereby reduced, making the results more easily understandable to the system user.

In this study, the research field was limited to artificial intelligence, and the set of keywords was assembled from the titles of presentations at the national conventions of the Society for Artificial Intelligence. Nine hundred titles in all, presented from the 14th to the 17th conventions, were collected. Nine hundred sixty-four nouns and phrases contained in the titles were collected as keywords. The procedure for assembling the nouns and phrases in the set of keywords was as follows.

[Step 1] Text files of the presentation titles from the 14 th to the 17th conventions were prepared.

[Step 2] The Chagama program [10] was applied to each text file. Keywords and nouns written in katakana form were extracted and were defined as the initial set of keywords.

[Step 3] For each keyword contained in the initial set of keywords, the number of titles containing that keyword was counted. If the frequency was equal to or greater than a threshold $T_{1}$, the keyword immediately before that keyword was listed. If the frequency of the listed keyword was equal to or greater than threshold $T_{2}$, a phrase was constructed by combining these two keywords.

[Step 4] The phrases and the initial set of keywords were combined and defined as a set of keywords in the database.

In this study, the thresholds were set as $T_{1}=5$ and $T_{2}$ $=2$. The reason for adding phrases to the set of keywords was as follows. It was desirable that the titles of the papers cover as many topics as possible, which made the keywords longer. When the Chagama program was applied to the titles, however, the obtained noun keywords were shorter. Since the keywords used in titles can also be obtained by connecting adjacent noun keywords, the phrases were included in the set of keywords. Table 1 shows part of the obtained keyword set.
Table 1. Examples of keywords in the database of papers' titles

\begin{tabular}{lll}
\hline Correlation rule & \multicolumn{1}{c}{ Mining } & \multicolumn{1}{c}{ Summary } \\
\hline Dialog & $\begin{array}{l}\text { Distributed } \\
\text { collaboration }\end{array}$ & Game \\
Management & Music & Net information \\
Community & Text & Database \\
Mobile robot & Internet & Model \\
\hline
\end{tabular}

Then, the number of titles in which each keyword in the keyword set appeared was counted. The results were stored in the database as the number of hits for that keyword at that time. Similarly, the number of titles in which two different keywords concurrently appeared was also stored in the database as the number of coincident hits on the two keywords.

\subsubsection{Diary database}

Convenience stores represent a food sector where new products, such as rice balls and lunch boxes, are continuously created. The fact that new products are created frequently implies a high possibility that a product will appear and succeed. Consequently, the new products to be predicted were restricted to foods, such as rice balls, lunch boxes, and teas. The number of hits on each keyword was estimated from the diaries of individuals appearing on the rental diary site "Sarusaru diary" [9].

The diary was utilized because it reflects the views of many users in daily life and has immediacy, making it easy to estimate keyword recognition accurately. Diaries of 5906 users, including 286,033 items, from March 2000 to May 2003, were used in the experiment. A total of 2096 nouns representing foods contained in the diary were used as keywords. The procedure for preparing the set of keywords was as follows.

[Step 1] For the diaries of users who wrote for at least a month in "Sarusaru diary" in or after March 2000, the HTML source was downloaded from March 2000 to May 2003.

[Step 2] The diary was divided by years and months. The text for the diary part was extracted by deleting HTML tags.

[Step 3] The Chagama program was applied to the extracted part. From the obtained nouns, keywords representing foods were selected manually and the result was defined as the keyword set.

Table 2 shows part of the obtained keyword set. 
Table 2. Examples of keywords in the database of diaries

\begin{tabular}{lll}
\hline Pudding & Melon & Yogurt \\
Wine & Pancake & Meat bun \\
Salad & Powdered tea & Cream \\
Hamburger & Raisins & Waffle \\
Oolong tea & Green tea & Cola \\
\hline
\end{tabular}

Then the diaries were divided into periods of 3 months from March 2000. The number of diaries in which each keyword in the keyword set appeared was counted, and the result was stored in the database as the number of hits on that keyword at that time. The number of diaries in which two different keywords both appeared was similarly stored as the number of coincident hits.

\subsection{Originality evaluator}

This section describes the procedure for evaluating the originality of combinations and gives the formula used to derive the evaluation score. Suppose that there are two keywords $L$ and $C$ forming a combination. Let the probabilities of these keywords be $P(L)$ and $P(C)$, respectively. The total number of data is denoted as all, and the number of data that contain keyword is denoted as hit(keyword). The probability of occurrence of keyword is defined as follows:

$$
P(\text { keyword })=\frac{\text { hit }(\text { keyword })}{\text { all }}
$$

If two keywords $L$ and $C$ appear independently, the probability that the keywords appear coincidentally is $P(L) P(C)$. There is a discrepancy, however, between $P(L) P(C)$ and the probability $P(L \cap C)$ that $L$ and $C$ appear coincidentally. The combination can be considered more original if $P(L \cap$ $C$ ) is smaller than $P(L) P(C)$. Consequently, the originality of the combination is evaluated as follows ${ }^{\dagger}$ :

$$
\operatorname{ori}(L, C)=\frac{P(L) P(C)}{P(L \cap C)}
$$

A combination is considered more effective if the originality value given by Eq. (2) decreases with time. The reason is that the decrease in originality indicates a tendency to spread widely. This situation is represented as follows:

$$
t_{\_} \text {ori }(L, C)=\frac{\text { ori }(L, C)-\text { ori }_{\text {init }}(L, C)}{y e a r}
$$

\footnotetext{
${ }^{\dagger}$ This corresponds to the mutual information.
}

Here ori $_{\text {init }}$ is the earliest value of ori in the data, and year is the number of years down to that oldest item of data.

\subsection{Novelty evaluator}

When a keyword has novelty, the rate of increase of the number of hits on that keyword is larger than that on keywords without novelty. Novelty is evaluated by the behavior of the number of hits on the keywords of the category extension side, as follows:

$$
t_{-} n o v(C)=\frac{h i t(C)-h_{i n i t}(C)}{y e a r}
$$

Furthermore, the novelty is higher for keywords with a smaller number of hits at the time of evaluation, even if the rate of increase is the same, in the sense that recognition will increase and the object will start to become popular. Consequently, the novelty of the keywords on the category extension side is evaluated by the equation

$$
\operatorname{nov}(C)=\operatorname{hit}(C)
$$

\subsection{Evaluation score calculator}

The evaluation score calculator aggregates four evaluation values provided by the originality evaluator and the novelty evaluator, and determines the comprehensive evaluation score for the combination. The comprehensive evaluation score is the sum of the evaluation values, normalized on the basis of their magnitude ranks. It is expressed as follows, where $\operatorname{Rank}()$ is a function that returns the order of the evaluation values:

$$
\begin{aligned}
& \text { evaluation }(L, C) \\
& =\operatorname{Rank}(\operatorname{ori}(L, C))+\operatorname{Rank}\left(t_{-} \text {ori }(L, C)\right) \\
& \quad+\operatorname{Rank}(\operatorname{nov}(C))+\operatorname{Rank}\left(t_{-} \operatorname{nov}(C)\right)
\end{aligned}
$$

The system outputs the keywords of the category extension side in decreasing order of the value of evaluation. The output is limited by the number of hits according to the following conditions. A keyword is not displayed if any of the following conditions is satisfied.

(1) The number of coincident hits on the keyword of the line extension side and the keyword of the category extension side is 0 in combination evaluation.

(2) The number of hits on the keyword of the category extension side is 0 in combination evaluation.

(3) Any of the evaluation values in Eqs. (2) to (5) is less than the average value.

Conditions (1) and (2) imply that there is now no combination with the keyword of the category extension side. The keyword is inadequate as output, since the com- 
bination implies a higher risk of original activity and has no indication of novelty. Condition (3) is intended to identify truly useful combinations by extracting only combinations that have high evaluation values for all aspects.

\section{Experiment}

This section describes the combination evaluation experiments that were performed for the two databases prepared for the paper and the diary. The purpose of the proposed system is to avoid situations in which the user attempting to engage in creation by combination exhaustively examines all combinations. For this purpose, we wish to present combinations which are highly likely to become popular, as useful material to aid creative activity. The details of the evaluation experiment for each database are presented below, together with a discussion.

\subsection{Prediction experiment for paper titles}

The data on the presentation titles at the 14th to 16th National Conventions of the Society for Artificial Intelligence were used, and combinations of keywords with increasing interest in the titles presented at the 17 th Convention were predicted, using the database described in Section 4.1.1. When the number of coincident hits continued to increase from the 15 th to the 17 th convention, it was decided that the interest in the combination had increased. Such a combination was judged to be a correct solution, and was compared with the system output. Increasing interest is a necessary condition for popularity.

Table 3 shows the keywords of the line extension side input to the system and the number of hits (number of titles containing the keyword) at each time. Table 4 shows the keywords on the category extension side, the keyword examples output by the system, and the combinations output by the system from 964 different combinations.

Table 5 shows the combinations in which interest increased from the 15 th to the 17 th convention, representing correct solutions of the experiment, together with

Table 3. Line keywords for prediction of papers' titles and their hit numbers

\begin{tabular}{llll}
\hline Line keyword & 14 th & 15 th & 16 th \\
Information & 17 & 21 & 23 \\
Knowledge & 21 & 16 & 21 \\
Discovery & 11 & 8 & 9 \\
Robot & 6 & 8 & 8 \\
Average of hit numbers & 1.2 & 1.8 & 1.9 \\
\hline
\end{tabular}

Table 4. Input and output keywords of this system in an experiment of prediction papers' titles

\begin{tabular}{llc}
\hline Input keyword & \multicolumn{1}{c}{$\begin{array}{c}\text { Examples of output } \\
\text { keywords }\end{array}$} & $\begin{array}{c}\text { Output } \\
\text { numbers }\end{array}$ \\
\hline Information & $\begin{array}{l}\text { Knowledge, multi, } \\
\text { interface }\end{array}$ & $97 / 964$ \\
Knowledge & Class, music, community & $59 / 964$ \\
Discovery & Pattern, model, chance & $24 / 964$ \\
Robot & Time, collaboration, & $23 / 964$ \\
& interactive & \\
\hline
\end{tabular}

the rank of each evaluation value. In the table, the system predicts all keywords on the category extension side to be included in the output. In fact, the combinations shown in Table 5 were observed in the titles of presentations at the 17th convention. Examples of the titles are "Fast Knowledge Discovery from XML Data Streams," [11] “Object Recognition from Actual Images for Autonomous Motion of Intelligent Robots," [12] and "Extraction and Information Support in Human-Related Networks on the Web" [13].

Table 5 contains combinations which could not be identified solely on the basis of the evaluation value of the novelty in the sense of likelihood of popularity. Examples are "knowledge and discovery" and "robots and intelligence." These were identified by including originality in the evaluation in order to represent innovative combinations as viewed by the public.

In this system, combinations were judged to be useful when the comprehensive score was high, even if the individual value was lower. That is, combinations effectively balancing novelty of the keywords on the category exten-

Table 5. Correct combinations and each evaluation value in an experiment of prediction papers' titles

\begin{tabular}{llrrrr}
\hline Line keyword & $\begin{array}{c}\text { Category } \\
\text { keyword }\end{array}$ & ori $(L, C)$ & $\begin{array}{c}t_{-} \text {ori } \\
(L, C)\end{array}$ & nov & $\begin{array}{r}t \_n o v \\
(L, C)\end{array}$ \\
\hline information & support & 20 & 605 & 1 & 1 \\
information & extraction & 20 & 585 & 34 & 1 \\
knowledge & discovery & 376 & 605 & 409 & 288 \\
discovery & data & 20 & 614 & 6 & 1 \\
discovery & knowledge & 20 & 602 & 4 & 1 \\
robot & movement & 400 & 398 & 317 & 82 \\
robot & intelligence & 391 & 389 & 420 & 93 \\
robot & operation & 409 & 409 & 295 & 82 \\
\hline
\end{tabular}


sion side and originality of the combination were judged to be useful. By using such an approach, combinations such as "knowledge and discovery" and "robots and intelligence," which actually became popular, were predicted, even though the individual evaluation values were low. In this sense, prediction by the proposed system combining evaluation values for novelty and originality is judged to have been a success.

On the other hand, the system outputs many keywords which are not included in Table 5. Table 6 shows the system outputs when "discovery" is input. In the table, the keywords other than "data" and "knowledge" are keywords which were not considered as correct solutions, since the number of coincident hits remained the same or decreased.

In the case of "rule," the number of coincident hits decreased. However, the originality was high. "Discovery of correlation rules" has recently attracted great interest in

Table 6. Output category keywords in inputting "discovering" and each evaluation value

\begin{tabular}{|c|c|c|c|}
\hline Output keyword & $\begin{array}{l}\text { Total of } \\
\text { values }\end{array}$ & $\begin{array}{l}\text { Originality } \\
\text { Eq. (2) + } \\
\text { Eq. (3) }\end{array}$ & $\begin{array}{c}\text { Novelty } \\
\text { Eq. (4) + } \\
\text { Eq. (5) }\end{array}$ \\
\hline rule & 38 & 3 & 35 \\
\hline pattern & 117 & 2 & 115 \\
\hline history & 258 & 65 & 193 \\
\hline electronics & 259 & 66 & 193 \\
\hline model & 357 & 343 & 14 \\
\hline order & 367 & 252 & 115 \\
\hline mining & 368 & 253 & 115 \\
\hline decision & 373 & 338 & 35 \\
\hline dialog & 392 & 304 & 84 \\
\hline summary & 392 & 304 & 84 \\
\hline knowledge & 660 & 623 & 27 \\
\hline data & 641 & 634 & 7 \\
\hline structure & 653 & 626 & 27 \\
\hline story & 1046 & 621 & 425 \\
\hline correlation & 1048 & 623 & 425 \\
\hline detection & 1049 & 624 & 425 \\
\hline view line & 1049 & 624 & 425 \\
\hline cascade & 1052 & 627 & 425 \\
\hline staying & 1052 & 627 & 425 \\
\hline chance & 1052 & 627 & 425 \\
\hline topic & 1052 & 627 & 425 \\
\hline compound & 1052 & 627 & 425 \\
\hline patch board & 1052 & 627 & 425 \\
\hline analog & 1052 & 627 & 425 \\
\hline
\end{tabular}

the data mining field. Thus, it is highly conceivable that there will be original activity focusing on this topic. However, the system output is expected to include combinations which first show significant popularity and then tend to decay. Such combinations will disappear in the future, with a decrease in the number of associated research topics.

For keywords other than "knowledge," "data," and "rule," the number of coincident hits remained the same. Although the tendencies of these keywords in the future are unknown, they were output as keywords satisfying the necessary conditions for popularity. For example, "chance discovery" and "topic discovery" are emerging topics that have been attracting attention. However, their future staying power is unknown. These outputs do not meet the sufficient condition for popularity, and the user must examine the outputs for usefulness. However, it is true that the system greatly restricts the number of combinations compared to the case in which the user must select useful combinations from all possible combinations. In fact, for the case of "discovery," the combinations were reduced from 2/964 to $2 / 24$ in the correct solution obtained in this experiment.

It is evident from Tables 3 and 4 that the system outputs a larger number of combinations when the number of hits on the keyword of the line extension side is higher. It is inferred that a larger number of combinations are attracting attention if the recognition of the keywords on the line extension side is increased. Thus, it is difficult to find truly useful combinations.

\subsection{Prediction experiment for new products}

Based on the text data in the diaries from June 2002 to February 2003, combinations of food materials used in new products in convenience stores that first went on sale between March and May 2003 were predicted by using the database described in Section 4.1.2. The combinations on which the number of coincident hits continued to increase from June 2002 to May 2003 were defined as correct solutions, and the results were compared to the system output.

Suppose that a new product first went on sale around March 2003. No combination constituting a correct solution is contained in that product, and the number of coincident hits is smaller. When the product first goes on sale, it draws the attention of many users, and the number of coincident hits increases. That is, a combination for which the number of coincident hits increases is considered as a combination that has begun to be popular. The performance of the system is estimated by comparing such combinations with the system output.

Table 7 shows the keywords of the line extension side input to the system and the number of hits at each time. Table 8 shows examples of the keywords output by the 
Table 7. Line keywords for prediction of new items and their hit numbers

\begin{tabular}{lccc}
\hline Line keyword & $2002 / 6$ & $2002 / 9$ & $2002 / 12$ \\
\hline rice ball & 29 & 32 & 28 \\
ramen & 112 & 113 & 99 \\
tea & 113 & 143 & 127 \\
\hline Average of hit numbers & 31 & 35 & 33 \\
\hline
\end{tabular}

system for each keyword of the line extension side and the number of combinations output by the system from all 2096 combinations.

Table 9 shows the system output (correct solutions are underlined) when "rice ball" is input as the keyword of the line extension side. For this case, Table 10 shows the combinations for which the number of coincident hits with "rice ball," representing the correct solution in this experiment, actually increased. Among 14 combinations for which the number of coincident hits increased, the system was able to predict 9, that is, "soba," "tsukune (bird-meat ball)," "ice-cream," "curry," "sandwich," "sea bream," "beans," "bean curd," and "miso soup" (underlined keywords in the table).

The reason for the prediction of these nine combinations is as follows. The combinations do not have a high evaluation value in originality alone or in novelty alone, but instead have fairly high evaluation values in both. Thus, the system outputs the combinations as useful by considering the two evaluation values in combination. Actual examples of products are "rice ball sandwich," "tsukune rice ball," and "sea bream rice." As another example, a product in which rice balls are used as dumplings in a mixture of miso soup and green tea appeared on the market in May 2003 in the "rice ball series" of Lawson Stores. It is evident from this case that the user should extract his or her own ideas

Table 8. Input and output keywords of this system in an experiment of prediction papers' titles

\begin{tabular}{llc}
\hline Input keyword & \multicolumn{1}{c}{$\begin{array}{c}\text { Examples of output } \\
\text { keywords }\end{array}$} & $\begin{array}{c}\text { Output } \\
\text { numbers }\end{array}$ \\
\hline rice ball & $\begin{array}{l}\text { eel, tsukune, carrot, fried } \\
\text { egg, potato chips } \\
\text { potato, chicken, bread, } \\
\text { chahan, onion, meat bun } \\
\text { ramen }\end{array}$ & $24 / 2096$ \\
tea & $\begin{array}{l}\text { ice cream, cake, tomato, } \\
\text { bean, salt, waffle }\end{array}$ & $52 / 2096$ \\
\hline
\end{tabular}

Table 9. Output category keywords in inputting "rice ball" and each evaluation value

\begin{tabular}{lccc}
\hline $\begin{array}{l}\text { Output key- } \\
\text { word }\end{array}$ & $\begin{array}{c}\text { Total of } \\
\text { values }\end{array}$ & $\begin{array}{c}\text { Originality } \\
\text { Eq. (2) + Eq. } \\
(3)\end{array}$ & $\begin{array}{c}\text { Novelty Eq. } \\
\text { (4) + Eq. (5) }\end{array}$ \\
\hline eel & 402 & 158 & 244 \\
$\underline{\text { tsukune }}$ & 407 & 241 & 166 \\
carrot & 421 & 202 & 219 \\
fried egg & 427 & 178 & 249 \\
sea bream & 443 & 249 & 194 \\
mayonnaise & 478 & 261 & 217 \\
radish & 494 & 268 & 226 \\
potato & 500 & 279 & 221 \\
miso soup & 515 & 282 & 233 \\
corn & 564 & 248 & 316 \\
flakes & 768 & 217 & 351 \\
sandwich & 584 & 271 & 313 \\
milk & 615 & 294 & 321 \\
soba & 634 & 256 & 378 \\
bread & 647 & 280 & 367 \\
curry & 668 & 270 & 398 \\
ramen & 677 & 262 & 415 \\
pickles & 710 & 231 & 479 \\
juice & 712 & 299 & 413 \\
potato chips & 713 & 141 & 572 \\
ice cream & 716 & 294 & 422 \\
$\underline{\text { beans }}$ & 724 & 281 & 443 \\
$\underline{\text { bean curd }}$ & 734 & 282 & 452 \\
bonito flakes & 1030 & 158 & 872 \\
\hline
\end{tabular}

from combinations in order to make creative activity a success, rather than directly combining food materials.

In Table 10, the system did not predict five keywords, "ginger," "beer," "seaweed," "soy sauce," and "natto," even though the number of coincident hits increased. The number of coincident hits on these keywords tends to increase and decrease periodically. For such keywords, the number of coincident hits was decreasing in the prediction stage, but increased from March to May 2003. The combinations predicted to become popular in the future on the basis of the past pattern of change of the number of coincident hits cannot be considered original, and are different from the useful combinations sought in this study. Consequently, it is not a problem that the system does not predict such combinations. 
Table 10. Correct combinations with "rice ball"

\begin{tabular}{lccc}
\hline $\begin{array}{l}\text { Category } \\
\text { keyword }\end{array}$ & $\begin{array}{c}\text { Total of } \\
\text { values }\end{array}$ & $\begin{array}{c}\text { Originality } \\
\text { Eq. (2) + Eq. } \\
(3)\end{array}$ & $\begin{array}{c}\text { Novelty Eq. } \\
(4)+\text { Eq. (5) }\end{array}$ \\
\hline ginger & 3202 & 1367 & 1835 \\
soba & 634 & 256 & 378 \\
tsukune & 407 & 241 & 166 \\
ice cream & 716 & 294 & 422 \\
curry & 668 & 270 & 398 \\
sandwich & 584 & 271 & 313 \\
beer & 2919 & 1367 & 1552 \\
seaweed & 3102 & 1380 & 1722 \\
soy sauce & 3251 & 1529 & 1722 \\
sea bream & 443 & 249 & 194 \\
$\underline{\text { beans }}$ & 724 & 281 & 443 \\
bean curd & 734 & 282 & 452 \\
natto & 2610 & 1529 & 1081 \\
$\underline{\text { miso soup }}$ & 515 & 282 & 233 \\
\hline \hline
\end{tabular}

In Table 9, for 15 keywords included in the system output, the number of coincident hits did not increase. Among these, the number of coincident hits decreased for 3, "mayonnaise," "potato," and "ramen." The system output these 3 keywords as useful combinations because the evaluation value for the novelty of the keyword on the category extension side and the evaluation value for the originality of the combination were both high. However, these combinations were not actually realized, and subsequently the number of coincident hits started to decrease and disappeared. It is interpreted that the combinations did not attract attention at all, or that they were noticed by some users but were not very noteworthy in realization. In other words, they were considered to be keywords incapable of achieving popularity.

On the other hand, there are keywords for which the number of coincident hits neither increased nor decreased. Examples of such keywords are "eel," "fried egg," "pickles," "potato chips," and "bonito flakes." Among these, for "eel" and "fried egg," the number of incidental hits neither increased nor decreased, including the period from March 2000 to February 2003, which was not used in the evaluation of combinations. Consequently, related combinations may emerge in the future. For "pickles," "potato chips," and "bonito flakes," on the other hand, the number of coincident hits neither increased nor decreased in the period from June 2002 to March 2003 used for the evaluation, but tended to decrease from March 2000 to June 2002.
At present, however, the amount of data to be used in the evaluation is determined empirically. Thus, the situations are the same for the evaluation functions between the members of the group of "eel," "fried egg" and the members of the group of "pickles," "potato chips," "bonito flakes." The latter group should not be included in the system output. Consequently, the evaluation expression should be improved to express more flexibly and accurately the temporal changes in the amount of data used for evaluation.

Table 11 shows the number of trials for each keyword of the line extension side until the combination constituting the correct solution was found, for the cases with and without system output. When there was no system output, the novelty had to be examined for all of 2096 combinations. One hundred fifty trials were needed on average until the combination constituting the correct solution was included. When the combinations to be examined were restricted by the system, on the other hand, the combination constituting the correct solution was included after 2 or 3 trials on average.

The greatest advantage in the use of the proposed system is that the number of trials until a combination with novelty is identified for examination can be reduced on average. Consider, for example, the case in which combinations are to be selected in order to develop a new product. If the system is not used, it is necessary to examine the novelty of all conceivable combinations, and a large number of trials are required until a combination which actually has novelty is found. If the system is used, on the other hand, combinations which actually have novelty are included in the output after a smaller number of trials. In this sense, the proposed system is very useful in the discovery of combinations with novelty.

\subsection{Comparison with time-series prediction by ARMA model}

Using an autoregressive moving average (ARMA) model [14], useful combinations were predicted by timeseries prediction from the number of coincident hits. The accuracy of the above approach and the accuracy of the proposed evaluation expression for combinations proposed

Table 11. Trial times average of finding correct combinations

\begin{tabular}{lcc}
\hline Line keyword & $\begin{array}{c}\text { Without system } \\
\text { outputs }\end{array}$ & $\begin{array}{c}\text { With system } \\
\text { outputs }\end{array}$ \\
\hline rice ball & 150 & 2 \\
ramen & 140 & 3 \\
tea & 116 & 3 \\
\hline
\end{tabular}


in this paper are compared experimentally in this section. The ARMA model is a model for time-series analysis in which the AR model and MA model are combined. It is used in the description of numerical data varying with a certain regularity, such as time series of stock prices and exchange rates.

Generally, for the set of time-series data $\left\{y_{t}\right\}$, the $p$-th order $\operatorname{AR}(p)$ model is represented as follows, where $k_{A R}$ and $\left\{a_{p}\right\}$ are constants and $u_{t}$ is the error term:

$$
y_{t}=k_{A R}+a_{1} y_{t-1}+a_{2} y_{t-2}+\cdots+a_{p} y_{t-p}+u_{t}
$$

The $q$-th order $\operatorname{MA}(q)$ model for $\left\{y_{t}\right\}$, on the other hand, is represented as follows, where $k_{M A}$ and $\left\{b_{q}\right\}$ are constants:

$$
y_{t}=k_{M A}+u_{t}+b_{1} u_{t-1}+b_{2} u_{t-2}+\cdots+b_{q} u_{t-q}
$$

In this experiment, the ARMA $(2,2)$ model represented below was used:

$$
y_{t}=k_{A R M A}+a_{1} y_{t-1}+a_{2} y_{t-2}+u_{t}+b_{1} u_{t-1}+b_{2} u_{t-2}
$$

Here $k_{A R M A}$ is a constant.

The same experiment as in Sections 5.1 and 5.2 was performed. In the ARMA model, the number of coincident hits on the keywords of the line category extension side and the keywords of the category extension side composing the combinations was used as the time-series data. The outputs from the ARMA model were defined as the same number of top-ranking items as in the proposed system. Table 12 shows the recall rate (the ratio of the number of predicted combinations to the number of combinations constituting correct solutions) in the prediction of paper titles. Table 13 shows the recall rate in the prediction of new products. It is evident that a better recall rate was achieved by the proposed method for both predictions.

The reason for the better recall rate is as follows. For cases in which the increase rate from the 14th to the 16th convention was not very large, or for cases in which the increase rate changed to a decrease at the 17 th convention, even if the increase rate was high from the 14th to the 16th,

Table 12. Recall comparison between ARMA model and this system in prediction papers' titles

\begin{tabular}{lcc}
\hline Line keyword & ARMA model & This system \\
\hline information & $1 / 2$ & $2 / 2$ \\
knowledge & $0 / 1$ & $1 / 1$ \\
discovery & $2 / 2$ & $2 / 2$ \\
robot & $1 / 3$ & $3 / 3$ \\
\hline
\end{tabular}

Table 13. Recall comparison between ARMA model and this system in prediction of new items

\begin{tabular}{lcc}
\hline Line keyword & ARMA model & This system \\
\hline rice ball & $5 / 14$ & $9 / 14$ \\
ramen & $6 / 16$ & $14 / 16$ \\
tea & $6 / 18$ & $13 / 18$ \\
\hline
\end{tabular}

it is difficult to make a prediction based only on the increase rate. On the other hand, the proposed method predicts combinations with an emphasis on the rarity of the combination by evaluating its originality, in addition to the rate of increase in the number of hits. Thus, it can be seen that originality must be evaluated in the creative activity of conceiving a new object, such as in the search for new research topics and the development of new products.

Thus, the recall rates obtained by the proposed four evaluation expressions [Eqs. (2) to (5)] and the recall rate obtained by Eq. (6) were compared as shown in Table 14. Comparing the number of predicted items, it can be seen that the number of predicted items is smaller if only an evaluation expression is applied. It is concluded, for the following reasons, that all of these evaluation expressions are necessary in the search of useful combinations.

Different combinations are predicted by different evaluation expressions. Equations (2) and (3) evaluate combinations based on the temporary value and the temporal behavior, respectively, of the originality. Equations (4) and (5) evaluate combinations on the basis of the temporary value and the temporal behavior, respectively, of the creativity of the keyword of the line extension side. Equations (2) and (5) evaluate the rarity of keywords. Equations (3) and (4) evaluate the frequency of appearance of keywords. Thus, the roles of the combination expressions are clearly divided.

Table 14. Recall comparison among four evaluation formula and proposed formula

\begin{tabular}{lccccr}
\hline $\begin{array}{l}\text { Line } \\
\text { keyword }\end{array}$ & Eq. (2) & Eq. (3) & Eq. (4) & Eq. (5) & Eq. (6) \\
\hline rice ball & $2 / 14$ & $2 / 14$ & $5 / 14$ & $6 / 14$ & $9 / 14$ \\
ramen & $3 / 16$ & $3 / 16$ & $8 / 16$ & $7 / 16$ & $14 / 16$ \\
tea & $2 / 18$ & $2 / 18$ & $4 / 18$ & $5 / 18$ & $13 / 18$ \\
\hline
\end{tabular}




\section{Conclusions}

This paper has considered objects created in the world as approximate combinations of existing items, and has proposed an evaluation system which discovers original combinations with a high probability of establishing themselves in the world. The proposed system estimates the temporal course of recognition of keywords by users and the temporal course of the novelty of combinations, and outputs combinations which are highly likely to establish themselves in the future.

The system outputs a set of keywords but does not state how the outputs should be utilized. The user must select combinations matched to his or her unique feelings and preferences from the narrowed range of choices that has output by the system. Then, the user must further analyze the selected item and perform creative activity. Considering that only humans can engage in a truly intellectual process, we have constructed a system that provides combinations which are useful as material to be utilized in the human thought process.

In the future, we plan to improve the evaluation expression so as to better express the actual changes. We also intend to consider combinations of three or more keywords. Combination of three or more keywords will be considered as follows. Assuming that a combination composed of two keywords has a certain probability of establishing itself, such a combination can be considered as a new keyword of the category extension side in the future.

We also plan to predict future success as seen from the future. Prediction of the future as seen from the future will be useful in the following context. When creative activity is aimed at product development, it will provide guidelines regarding which products should be developed at the present stage. In the case of research activity, it will support the making of decisions as to which research topics should be emphasized at the present stage, and to what fields they should be connected.

\section{REFERENCES}

1. Kubota H, Nishida T. Generation of creative dialog by multiple agents based on past utterance of user. Trans IEICE 2001;J84-D-I:1222-1230.
2. Weizenbaum, J. Eliza-A computer program for the study of natural language communication between man and machine. Commun ACM 1966;9:36-43.

3. Takasugi K, Kunifuji S. Construction of thought support system for idea emergence using spring model. J Soc Artif Intell 1999;14:495-503.

4. Sunayama W, Yachida M. Discovery of interested keyword from Web to predict prevalence in future. $\mathbf{J}$ Japan Soc Intell Inf Fuzziness 2003;15:309-317.

5. Ikeda S, Kaneda S, Kanasugi T. A statistical study of prevailing word prediction using dictionary of concurrent terms. Tech Rep IEICE, Vol. 100, No. 541, 2001.

6. Osawa S, Yachida M. Support of dangerous dislocation search from earthquake history data by applying keyword extraction tool KeyGraph. J Soc Artif Intell 2000;15:665-672.

7. Osawa S, Benson N, Yachida M. Keyword extraction by decomposition and integration of co-occurrence graph KeyGraph of words. Trans IEICE 1999;J82-DI:391-400.

8. Brand strategy of small and middle sized companies. Report of Finance Corp. for Small and Middle Sized Companies, No. 2003-3, 2004.

9. Diary rental site, (URL) http://www.diary.ne.jp/

10. Matsumoto Y, Kitauchi K, Yamashita T, Hirano Y, Matsuda H, Asahara M. Chagama analysis system for Japanese mora. Version 2.0. Manual version 2, NAIST-IS-TR99012, (1999), (URL) http://chasen. aist-nara.ac.jp/index.html.ja

11. Asai T, Abe K, Kawazoe S, Arimura H, Arikawa S. Fast knowledge discovery from XML data stream, 17th Natl Conv Soc Artif Intell, 2002.

12. Higuchi Y, Hayashi K, Watabe K, Kawaoka T. Object recognition from actual images for autonomous motion of intelligent robots. 17th Natl Conv Soc Artif Intell, 2002.

13. Matsuo Y, Tomobe H, Hashida K, Ishibashi M. Extraction and information support in human-related networks on the Web. 17th Natl Conv Soc Artif Intell, 2002.

14. Tanaka T, Sasayama S, Sakagami T. Macro-economic analysis. Learning of economics from table calculation. Chuo Keizaisha Co.; 1995. 
AUTHORS (from left to right)
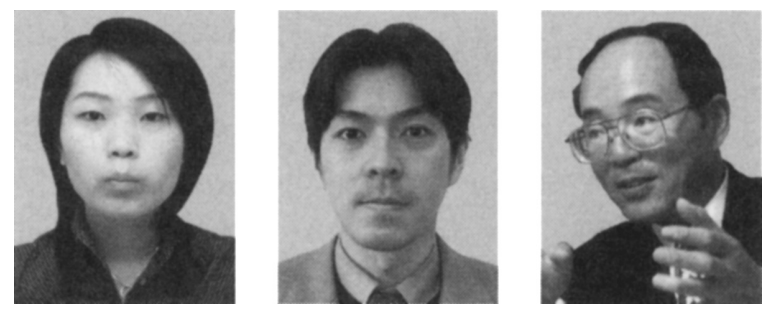

Yoko Nishihara received a B.S. degree from the Department of System Science, Osaka University, in 2003 and is now in the first half of the doctoral program.

Wataru Sunayama received a B.S. degree from the Department of Control Engineering, Osaka University, in 1995 and completed the first half of the doctoral program in 1997. While enrolled in the second half of the doctoral program, he accepted an appointment as a research associate in 1999. He has been an associate professor on the Faculty of Information Sciences, Hiroshima City University, since 2003. He holds a D.Eng. degree. He is engaged in research on support for human creative activity.

Masahiko Yachida completed the M.E. program at the Graduate School of Engineering, Osaka University, in 1971 and joined the Faculty of Engineering Science as a research associate, subsequently becoming an associate professor and a professor. Since 1997, he has been a professor in the Graduate School of Engineering Science. He holds a D.Eng. degree. He is engaged in research on image processing, artificial intelligence, and mobile robots. His publications include Robot Vision (Shokodo) and Computer Vision (Maruzen, author and editor). He is a member of the Information Processing Society and the Robotics Society of Japan. 